\title{
Electrostriction in field-structured composites: Basis for a fast artificial muscle?
}

\author{
James E. Martin and Robert A. Anderson \\ Sandia National Laboratories \\ Albuquerque, NM 87185-1421
}

\begin{abstract}
The electrostriction of composites consisting of dielectric particles embedded in a gel or elastomer is discussed. It is shown that when these particles are organized by a uniaxial field before gelation, the resulting field-structured composites are expected to exhibit enhanced electrostriction in a uniform field applied along the same axis as the structuring field. The associated stresses might be large enough to form the basis of a polymer-based fast artificial muscle.
\end{abstract}

Sandia is a multiprogram laboratory operated by Sandia Corporation, a Lockheed Martin Company, for the United States Department of Energy under contract DE-AC04-94AL8500. This work supported by the Division of Materials Sciences, Office of Basic Energy Sciences, U.S. Department of Energy (DOE). 


\section{DISCLAIMER}

This report was prepared as an account of work sponsored by an agency of the United States Government. Neither the United States Government nor any agency thereof, nor any of their employees, make any warranty, express or implied, or assumes any legal liability or responsibility for the accuracy, completeness, or usefulness of any information, apparatus, product, or process disclosed, or represents that its use would not infringe privately owned rights. Reference herein to any specific commercial product, process, or service by trade name, trademark, manufacturer, or otherwise does not necessarily constitute or imply its endorsement, recommendation, or favoring by the United States Government or any agency thereof. The views and opinions of authors expressed herein do not necessarily state or reflect those of the United States Government or any agency thereof. 


\section{DISCLAIMER}

Portions of this document may be illegible in electronic image products. Images are produced from the best available original document. 


\section{Introduction}

When a suspension of dielectric particles is exposed to a uniaxial electric field, the induced dipole moments will cause the particles to chain along the field lines to form complex anisotropic structures, providing dielectric contrast exists between the particle and liquid phase. These structures greatly modify the shear rheology of the suspension and are the basis for the well-known electrorheological effect. [1] If the liquid phase is polymerized while the field is applied, these anisotropic structures can be trapped to form field-structured composites. These composites have anisotropic properties, some of which have been reported in the literature, including the conductivity and permittivity, $[2,3]$ dielectric breakdown field, [4] and optical transmittance. [5, 6] In this paper we discuss the enhanced electrostriction that field-structured materials exhibit, and the possibility of using these materials as fast artificial muscles. We will show that the expected electrostriction of these materials has a large contribution from the induced dipoles on the particles interacting with each other.

In fact, two types of field structured composites have been demonstrated. In addition to the uniaxial composites, of which a sample is shown in Fig. 1, it is possible to create biaxial composites structured in a biaxial field, such as a rotating field. When a rotating field is applied in the $\mathrm{x}-\mathrm{y}$ plane at a sufficiently high frequency that particles do not move much in one period, an average dipolar interaction is created between particles that is exactly $-1 / 2$ the dipolar interaction produced by a uniaxial field applied along the $\mathrm{z}$ axis. [7] The result of this is the formation of plates in the $x-y$ plane, as shown in Fig. 1 for a sample of magnetic particles that was physically rotated between fixed magnets.

These uniaxial and biaxial structures form when a field is applied, in order to reduce the free energy of the composite and its attached power supply, and a large contribution to this is the net electrostatic energy. This energy is reduced when the dipole-dipole interaction energy is reduced, a consequence of which is an increase of the effective dielectric constant of the composite. Because field-structured composites (FSCs) form to minimize the electrostatic energy, one might surmise that the electrostatic energy might increase rapidly with deformation of these materials, and that the dielectric constant might decrease rapidly. A major contribution to the electrostriction effect is 
related to the derivative of the dielectric constant with respect to strain and therefore FSCs should exhibit large electrostrictive effects.

Before computing the electrostrictive effect, it is useful to develop an intuitive understanding of electrostriction in FSCs. First consider a chain of high permittivity, hard spherical particles in a liquid. When an electric field is applied along the chain direction, dipole moments will be induced on the particles which will create attractive interactions between them. These attractive interactions will create positive pressure on the particles and will be balanced by the mechanical deformation of the particles, causing them to become very slightly oblate. If one were to apply a tensile force to this chain, by literally pulling on opposite ends, one would find that the chain would pull apart when the strain is just large enough that small gaps start to form between the spheres. The reason for the small yield strain is that the electrostatic interaction, being a solution to Laplace's equation, has negative curvature. Because of the negative curvature, the interaction of a single sphere with its two slightly parted neighbors is bistable, Fig. 2, having minima when the central sphere is in contact with either of its neighbors, and a maximum when equidistant from its neighbors. Thus if one were to affinely stretch a chain of dipoles and could somehow arrange that all were equidistant, the slightest amount of thermal motion would cause gap coalescence, and thus the breaking of the chain. [8] If the spheres are very hard, and they usually are, the work needed to break a chain would be extremely small.

This instability should be ameliorated when the chain is embedded in a relatively soft gel. The surrounding gel contributes an effective interaction between particles that should stabilize the stretching of a chain, so that the particle dipole contribution to work of extension is large. In fact, affine deformation of the FSC should be a good approximation, even though it is completely incorrect when the suspending medium is a liquid.

Another aspect of FSCs is the detailed mechanics of deformation. The particles in an FSC would typically be much harder than the gel phase, so that in deformation virtual stress singularities occur in the gel phase at particle contacts. We expect these regions of the gel will rupture when the material is worked, so that a certain work softening occurs. If the FCS were first formed in a gel, and the gel was then swollen slightly, we would 
expect these particle contact regions to break, and that the particle centers would move affinely in the swollen gel, creating separations such that the electrostrictive stress would lead to a substantial deformation of the gel as particles were drawn together by the induced dipoles. This is probably the most practical method of producing an electrostrictive FSC, but perhaps a better method would be to coat the dielectric particles with a soft gel coating, whose shear modulus was at least as small as that of the gel phase. The FSC would then form with the dielectric particles neatly spaced.

One advantage electrostrictive FSCs would have over so-called electromotive gels [9] is the very rapid response, which is scale independent. The creation of stress in an FSC would be limited by the polarization time of the particles, and if the particles have a large intrinsic polarizability, such as $\mathrm{BaTiO}_{3}$, this time would be extremely fast. Electromotive gels are limited by the diffusion of ions and water through the gel, and this is slow and scales as the square of the size of the gel. Ionic gels are also mechanically weak, whereas an FSC could be made in a strong elastomer.

\section{Theory}

Approach: We are interested in computing the electrostriction of a composite material consisting of particles of volume fraction $\phi$ embedded in an elastomer or gel. When a uniform field is applied to this material through contacting electrodes, there will be two primary sources of electrostriction: the mutual attraction of the electrodes due to their free charge density; and the mutual interaction of the induced dipole moments on the particles. The electrodes have a mutual attraction because a uniform field exerts a force on a monopole. A field gradient exerts a force on a dipole, so the dipoles only interact with each other.

We follow the method of Anderson, [10] who computed the electrostriction of a simple cubic lattice of point dipoles, showing that the classical Maxwell stress, which is linear in the dielectric constant, is only one contribution to the total stress, which contains terms quadratic in the dielectric constant. To compute the total stress one must consider the dependence of the dielectric constant on strain. We will use this approach to 
compute the electrostriction effect of disordered materials such as field structured composites.

In the method of Anderson the electrostatic free energy is minimized. The electrical free energy in a capacitor of capacitance $\mathrm{C}$ is

$$
F_{e}=-C V^{2} / 2
$$

which includes the energy stored in the voltage source. The capacitance is

$$
C=\kappa_{3} \varepsilon_{0} A / d
$$

where $\kappa_{3}$ is the dielectric constant of the dielectric in the direction of the applied field (zaxis), $\varepsilon_{0}$ is the vacuum permittivity, $A$ is the lateral area of the capacitor, and $d$ is the electrode spacing.

The uniaxial electrostriction coefficients can be defined through the dependence of the dielectric constant on the tensile strain components $s_{i}$,

$$
\kappa_{3}=\kappa\left(1-\sum_{i=1}^{3} \gamma_{3 i} s_{i}\right)
$$

and can be expressed as $\gamma_{3 i}=-\kappa^{-1} \partial \kappa_{3} / \partial s_{i}$. Including the change in the capacitance due to changes in the dielectric area and thickness gives, to first order in strain, for the electrostatic free energy density

$$
\frac{1}{A d} F_{e}=-\frac{\kappa \varepsilon_{0} E_{0}^{2}}{2}\left(1+s_{1}+s_{2}-s_{3}-\sum_{i} \gamma_{3 i} s_{i}\right)
$$

The tensile stresses $\sigma_{i}$ are obtained by differentiating the electrostatic free energy density with respect to the strains, $\sigma_{i}=\frac{-1}{A d} \frac{\partial F_{e}}{\partial s_{i}}$, giving 


$$
\begin{gathered}
\sigma_{1}=\frac{\kappa \varepsilon_{0} E_{0}^{2}}{2}\left(1-\gamma_{31}\right) \\
\sigma_{3}=-\frac{\kappa \varepsilon_{0} E_{0}^{2}}{2}\left(1+\gamma_{33}\right)
\end{gathered}
$$

Anderson [10] has pointed out that the quantities in the parentheses are corrections to the Maxwell stress, and these corrections are due to the dependence of the dielectric constant on strain. If the terms in the parentheses are positive, there is a compressive stress along the applied field and a tensile strain in the orthogonal directions. (In the materials of interest here, the $\mathrm{x}$ and $\mathrm{y}$ directions are equivalent, so $\sigma_{1}=\sigma_{2}$.) Anderson has also shown that for the cubic lattice this approach is equivalent to summing the forces of interaction between the dipoles and electrodes across a plane orthogonal to the axis of a stress component.

Particles in a dielectric continuum: In this paper we are concerned with computing the electrostrictive effect that is due to the presence of particles of dielectric constant $\kappa_{p}$ in a dielectric continuum of dielectric constant $\kappa_{c}$. To simplify the calculation, we make the following observation: The lines of force of the electric field $\mathbf{E}$ in any given composite structure will only depend on the ratio $\alpha=\kappa_{p} / \kappa_{c}$, and thus can be computed correctly by setting $\kappa_{c}^{\prime}=1$ and $\kappa_{p}^{\prime}=\alpha$. The electrostatic energy density, however, is just $\frac{1}{2} \mathbf{D} \cdot \mathbf{E}$ and using $\kappa_{c}^{\prime}=1$ and $\kappa_{p}^{\prime}=\alpha$ will lead us to a displacement field $\mathbf{D}^{\prime}$ that is reduced from the correct displacement field $\mathbf{D}$ by the factor $\kappa_{c}$. Thus the true energy density will be $\frac{1}{2} \mathbf{D} \cdot \mathbf{E}=\frac{1}{2} \kappa_{c} \mathbf{D}^{\prime} \cdot \mathbf{E}$, and the effective dielectric constant $\kappa_{e f f}^{\prime}$ computed with $\kappa_{c}^{\prime}=1$ and $\kappa_{p}^{\prime}=\alpha$ will be related to the true effective dielectric constant by $\kappa_{\text {eff }}=\kappa_{c} \kappa_{\text {eff }}^{\prime}$. Likewise, setting $\kappa_{c}^{\prime}=1$ and $\kappa_{p}^{\prime}=\alpha$ will underestimate the forces between particles by the factor of $\kappa_{c}$. Thus the electrostriction coefficients are computed correctly using $\kappa_{c}^{\prime}=1$ and $\kappa_{p}^{\prime}=\alpha$, but to get the stresses right one must use $\kappa_{\text {eff }}$ as the $\kappa$ that appears explicitly in Eqs. 
5a\&b. In the following we have set $\kappa_{c}^{\prime}=1$ and $\kappa_{p}^{\prime}=\alpha$, and denote the strain-dependent dielectric constant we compute as $\kappa_{\text {eff }, 3}^{\prime}$.

The local field: To compute the electrostriction coefficients we must first develop a relation for the dependence of the dielectric constant on strain. The dielectric constant is related to the susceptibility by $\kappa_{3}^{\prime}-1=\chi_{3}^{\prime} / \varepsilon_{0}$ where $\mathbf{P} \cdot \hat{\mathbf{z}}=\chi_{3}^{\prime} \mathbf{E}_{0} \cdot \hat{\mathbf{z}}$ and $\mathbf{P}$ is the polarization of the dielectric. The $\mathbf{z}$-component of the polarization is $\mathbf{P} \cdot \hat{\mathbf{z}}=\mathbf{m} \cdot \hat{\mathbf{z}} / \mathbf{v}$ where $\mathrm{v}=4 \pi a^{3} / 3 \phi$ is the volume per dipole in the unstrained state and $\mathbf{m} \cdot \hat{\mathbf{z}}=4 \pi a^{3} \varepsilon_{0} \beta \mathrm{E}_{l o c} \cdot \hat{\mathbf{z}}$, where $\mathbf{E}_{l o c}$ is the local field. Here the particle radius is $a$, the dielectric contrast factor is $\beta=(\alpha-1) /(\alpha+2)$, and the vacuum permittivity is $\varepsilon_{0}$. To compute the electrostriction coefficients we must therefore find the local field in a strained dielectric.

The local field at a dipole site is the sum of the field due to the free charge density on the electrodes and that due to all of the dipoles in the system. In the method of Lorentz, developed to simplify local field calculations, one instead adopts the perspective that the local field is the sum of the applied field, due to the free charge on the electrode plus the bound (polarization) charge on the dielectric at the electrode surface, and the field due to the nearby dielectric. The field due to the nearby dielectric is then the field due to the nearby dipoles, that reside in an imaginary cavity centered on the dipole of interest, and the Lorentz cavity field, which is due to the bound charge on the surface of the cavity (see Appendix A). The cavity can be of any shape and we choose a cubical cavity in the unstrained material. The local field is then $\mathbf{E}_{l o c}=\mathbf{E}_{0}+\mathbf{E}_{\text {dip }}+\mathbf{E}_{L C}$ where $\mathbf{E}_{0}$ is the applied field, $E_{d i p}$ is the field produced by the dipoles within the cavity, and $\mathbf{E}_{L C}$ is the field produced by the bound charge on the cavity.

If the Lorentz cavity is allowed to deform affinely with the applied strain, the zcomponent of the Lorentz cavity field can be written [10] (see Appendix B)

$$
\mathbf{E}_{L C} \cdot \hat{\mathbf{z}}=\frac{\mathbf{m} \cdot \hat{\mathbf{z}}}{3 \varepsilon_{0} \mathbf{v}}\left[1-\sum_{i} s_{i}+\left.\left(\frac{\sqrt{3}}{\pi}\right)\left(s_{1}+s_{2}-2 s_{3}\right)\right|_{]}\right.
$$


The first term on the rhs of Eq. 1 is the Lorentz cavity correction for a volume of cubic symmetry centered on the particle, and the term in square brackets is the effect of strain.

In the unstrained state the field at the $\mathrm{i}$-th dipole due to the nearby dipoles is

$$
\begin{gathered}
\mathbf{E}_{d i p} \cdot \hat{\mathbf{z}}=\frac{\mathbf{m} \cdot \hat{\mathbf{z}}}{2 \pi \varepsilon_{0} a^{3}}\left(-\psi_{2}\right) \\
\psi_{2}=-\sum_{j \neq i}\left(\frac{a}{r_{i j}}\right)^{3} \mathrm{P}_{2}\left(\cos \theta_{i j}\right)
\end{gathered}
$$

where $\mathrm{P}_{2}(x)=\frac{1}{2}\left(3 x^{2}-1\right)$ is the second Legendre polynomial, $\theta_{i j}$ is the angle the interparticle vector $\mathbf{r}_{\mathrm{ij}}$, of magnitude $r_{i j}$, makes to the applied field direction. The sum is over all other particles in the Lorentz cavity and is determined by the vector particleparticle pair distribution function. This field expanded in the strain is

$$
\mathbf{E}_{d i p}(\mathbf{s}) \cdot \hat{\mathbf{z}}=\mathbf{E}_{d i p}(\mathbf{0}) \cdot \hat{\mathbf{z}}+\left.\mathbf{s} \cdot \nabla_{s} \mathbf{E}_{d i p}(\mathbf{s}) \cdot \hat{\mathbf{z}}\right|_{s=\dot{0}}
$$

and if we now restrict our attention to materials in which the $\mathrm{x}$ and $\mathrm{y}$ axes are equivalent, and assume that all interparticle vectors deform affinely with strain, so $\mathbf{r}_{i j}=\left[x\left(1+s_{1}\right), y\left(1+s_{2}\right), z\left(1+s_{3}\right)\right]$, we obtain

$$
\begin{gathered}
\left.\mathbf{s} \cdot \nabla_{s} \mathbf{E}_{d i p}(\mathbf{s}) \cdot \hat{\mathbf{z}}\right|_{\mathbf{s}=0}=\frac{\mathbf{m} \cdot \hat{\mathbf{z}}}{2 \pi \varepsilon_{0} a^{3}} \frac{3}{7}\left[2\left(\psi_{2}-\psi_{4}\right)\left(s_{1}+s_{2}\right)+\left(3 \psi_{2}+4 \psi_{4}\right) s_{3}\right] \\
\psi_{4}=-\sum_{j \neq i}\left(\frac{a}{r_{i j}}\right)^{3} \mathrm{P}_{4}\left(\cos \theta_{i j}\right)
\end{gathered}
$$

where $P_{4}(x)=\frac{1}{8}\left(35 x^{4}-30 x^{2}+3\right)$ is the fourth Legendre polynomial.

The sum in Eq. 9b looks troublesome, because it is essentially a three dimensional integral over a integrand proportional to $r^{-3}$, thus threatening a logarithmic divergence. However, the potential logarithmic divergence will occur as $r$ goes to $\infty$, and in this limit 
the dielectric can be viewed as homogeneous. Thus if the angular integral $\int_{0}^{\pi} \mathrm{P}_{n}(\cos \theta) \sin \theta d \theta$ for $n=2,4$ vanishes, the divergence will not occur. In fact, for $n \geq 1$ this is true because of the orthogonality of Legendre polynomials on $(-1,1)$ : $\int_{0}^{\pi} \mathrm{P}_{n}(\cos \theta) \sin \theta d \theta=\int_{-1}^{1} \mathrm{P}_{0}(x) \cdot \mathrm{P}_{n}(x) d x=0$, where $\mathrm{P}_{0}(\mathrm{x})=1$.

The local field is found by combining Eqs. 6-9, and using $\mathbf{m} \cdot \hat{\mathbf{z}}=4 \pi a^{3} \varepsilon_{0} \beta \mathbf{E}_{l o c} \cdot \hat{\mathbf{z}}$, to obtain

$E_{l o c} \cdot \hat{z}=$

$E_{0} \cdot \hat{z}$

$\overline{\left\{1-\beta\left(\phi-2 \psi_{2}\right)-\beta \frac{6}{7}\left[2\left(\psi_{2}-\psi_{4}\right)\left(s_{1}+s_{2}\right)+\left(3 \psi_{2}+4 \psi_{4}\right) s_{3}\right]+\beta \phi\left[\sum_{i} s_{i}-\left(\frac{\sqrt{3}}{\pi}\right)\left(s_{1}+s_{2}-2 s_{3}\right)\right]\right\}}$

We emphasize that this expression is only valid for materials that are structurally equivalent along the $\mathrm{x}$ and $\mathrm{y}$ axes.

Electrostriction coefficients: The electrostriction coefficients can be obtained from the dielectric constant, which can be obtained from the polarization. Accounting for the dependence of the volume per dipole on strain, one obtains

$$
\mathbf{P} \cdot \hat{\mathbf{z}}=\varepsilon_{0}\left(\kappa_{e f f, 3}-1\right) \mathbf{E}_{0} \cdot \hat{\mathbf{z}}=3 \varepsilon_{0} \beta \phi\left(1-\sum_{i} s_{i}\right) \mathbf{E}_{l o c} \cdot \hat{\mathbf{z}}
$$

From Eqs. 10\&11 the value of $\kappa_{e f f, 3}$ can be obtained and the electrostriction coefficients can be obtained by differentiation, with the result

$$
\gamma_{3 i}=\frac{\left(\kappa_{e f f}^{\prime}-1\right)\left(\kappa_{e f f}^{\prime}+2\right)}{3 \kappa_{e f f}^{\prime}}-\lambda_{i} \frac{\left(\kappa_{e f f}^{\prime}-1\right)^{2}}{\kappa_{e f f}^{\prime}}
$$

where 


$$
\begin{aligned}
& \lambda_{1,2}=\frac{1}{\pi \sqrt{3}}+\frac{4}{7} \frac{\left(\psi_{2}-\psi_{4}\right)}{\phi} \\
& \lambda_{3}=\frac{-2}{\pi \sqrt{3}}+\frac{2}{7} \frac{\left(3 \psi_{2}+4 \psi_{4}\right)}{\phi}
\end{aligned}
$$

and the dielectric constant in the unstrained state is

$$
\kappa_{e f f}^{\prime}=\frac{1+2 \beta\left(\phi+\psi_{2}\right)}{1-\beta\left(\phi-2 \psi_{2}\right)}
$$

Dividing by the volume fraction in Eqs. 13a\&b looks troublesome, but this multiplies $\left(\kappa_{e f f}^{\prime}-1\right)^{2} \propto \phi^{2}$, so nothing horrible happens as $\phi \rightarrow 0$. For many structures, such as those with cubic symmetry, $\psi_{2}=0$ and $\lambda_{3}=-2 \lambda_{1,2}$. We now need only compute $\kappa_{\text {eff }}^{\prime}$ and the coefficients $\lambda_{i}$ from a model of the composite structure.

Field-structured composites: We have recently reported large-scale simulations of the evolution of structure of suspensions of spherical particles suddenly exposed to a uniaxial field along the z-axis, or a rotating field in the $x-y$ plane. [3] The uniaxial field causes the formation of chain-like structures such as those shown in Fig. 3, and the biaxial field causes the formation of sheet-like structures such as those shown in Fig. 3 . We will use these simulated structures to compute the electrostriction coefficients.

To compute the $\psi_{2,4}$ it is useful to define a vector pair correlation function for the cylindrical coordinates $z, \rho\left(\rho^{2}=x^{2}+y^{2}\right)$. Let $P(z, \rho) d z d \rho$ denote the probability that two particles are separated by a distance whose $z$ magnitude is within $[z, z+d z]$ and whose magnitude orthogonal to the $z$ axis is within $[\rho, \rho+d \rho]$, where the search domain around each particle is centered on the particle and is the size of the simulation volume, containing $\mathrm{N}$ particles. The normalization is then $\iint P(z, \rho) d z d \rho=N(N-1) / 2$. With this normalization, $r^{2}=\rho^{2}+z^{2}$, and $N>1$, 


$$
\psi_{k}=-N^{-1} \iint\left(\frac{a}{r}\right)^{3} P_{k}(\cos \theta) P(z, \rho) d z d \rho
$$

The case of randomly distributed particles is notable. Here the pair distribution function does not have an angular dependence, and because of the orthogonality of Legendre polynomials discussed above, $\psi_{2}=\psi_{4}=0$.

\section{Results and Discussion}

The results of these computations are shown in Table I for the simple cubic (SC) and body-centered tetragonal (BCT) lattices, as well as random particle dispersions, and uniaxial and biaxial field structured composites, all in the limit of large contrast, $\beta=1$. The values of $\psi_{2,4}$ are large for both the uniaxial and biaxial FSCs, and decrease with volume fraction, due to the reduced structural anisotropy at high concentration. Notable are the large values of $\kappa_{\text {eff }}^{\prime}$ for the BCT lattice - the ground state in a uniaxial field - and for the uniaxial FSCs. The dielectric constant for the biaxial FSCs is quite low along the $z$ axis.

Table II shows that the electrostriction coefficient along the $\mathrm{z}$ axis is much larger for the BCT lattice and uniaxial FSCs than for the random particle dispersions, as expected. Biaxial FSCs have very small electrostriction coefficients along both axes, indicating that the structure of these materials tends to cancel other corrections to the Maxwell stress.

We are more concerned with the particle-induced stresses, which we have normalized by $\sigma^{*}=\sigma / \frac{1}{2} \kappa_{c} \varepsilon_{0} E_{0}^{2}$. These stresses are in addition to the electrostrictive stresses expected for the unfilled polymer. The data in Table II show that these stresses are quite large along the $\mathrm{z}$ axis for the BCT lattice and uniaxial FSCs. At 30 vol. \% a uniaxial FSC has $3.5 \mathrm{x}$ the stress along the $\mathrm{z}$ axis as would a random particle dispersion. A surprising effect is that for the BCT lattice and uniaxial FSCs the stress along the $\mathrm{x}$ axis can actually be compressive, whereas for biaxial FSCs there is an enhanced expansion, due to the strong dipolar repulsion. 
How great is the electrostrictive stress due to the particles compared to that produced by the continuous phase alone? Let us take the example of a composite material of $30 \mathrm{vol} . \%$ particles of high dielectric contrast, $\beta=1$, in a polymer of dielectric constant $\kappa_{c}=5$. The z-axis stress in the unfilled polymer itself should be $-10.1 \varepsilon_{0} E_{0}^{2}$, assuming the material is random. If the particles are mixed in randomly, the additional stress arising from the particles will be $-11.9 \varepsilon_{0} E_{0}{ }^{2}$; if structured in a uniaxial field the particles will generate a stress of $-48.8 \varepsilon_{0} E_{0}{ }^{2}$. If one could contrive a BCT lattice of particles, the ground state for a field-structured composite, the particle stress would be $195 \varepsilon_{0} E_{0}{ }^{2}$. These computed particle stresses can be compared to those particle stresses expected for an electrorheological fluid, based on the point dipole approximation. An analytic theory we have developed for ER fluids [8] predicts that the stress in shear flow will be about $1.7 \varepsilon_{0} E_{0}{ }^{2}$ without considering local field effects (local field effects would increase this by a factor of $\sim 7-8$ but computer simulations show a stress $5 \mathrm{x}$ lower than this due to the formation of complex sheet-like structures that form in shear flow.) Thus we expect the electrostrictive effect to be much larger than that the ER effect.

If the material is allowed to strain, the elastic properties of the gel phase will determine the strain. To a good approximation we expect the gel to be incompressible, so that a compressive strain along the $\mathrm{z}$ axis will result in tensile strains along the $\mathrm{x}$ and $\mathrm{y}$ axes, with $s_{3}=-2 s_{2}=-2 s_{1}$. Thus the strain will be proportional to the net stress

$$
\sigma_{3}-\sigma_{1}=-\frac{1}{2} \kappa_{c} \kappa_{e f f} \varepsilon_{0} E_{0}^{2}\left[2+\left.\left(\frac{\sqrt{3}}{\pi}-\frac{2}{7} \frac{\psi_{2}+6 \psi_{4}}{\phi}\right) \frac{\left(\kappa_{e f f}^{\prime}-1\right)^{2}}{\kappa_{e f f}^{\prime}}\right|_{]}\right.
$$

The data in Table II show that the expected strain will in fact be compressive along the $\mathrm{z}$ axis for all structures studied, with large strains expected for lattices and uniaxial FSCs. Of course, the actual strains will be large only if the hard dielectric particles are separated by swelling or coating.

It is well known that the point dipole approximation underestimates the actual force of interaction between two vicinal particles when the dielectric contrast is large. [11-13] This is often referred to as a multipolar effect, but though correct, this 
terminology is somewhat misleading. Highly polarized spheres have the capping charge very close to the poles of the spheres, so that when two such spheres are brought into contact along the field axis, the ends of the dipoles on the two different spheres are almost brought into contact. The dipole-dipole interaction is still most of the interaction, but the simple point dipole approximation underestimates this. In any case, the actual particle stresses in particle composite materials of high dielectric contrast should be considerably larger than the point dipole approximation prediction contained herein. This is especially true for FSCs, where the particles have been brought into close proximity.

\section{Conclusions}

We have shown that uniaxial field structured composites consisting of high permittivity particles in a gel or elastomer should exhibit large electrostrictive stresses, compared to random particle dispersions, or the gel itself. We have computed the electrostrictive stresses in the point dipole approximation, and expect the measured stresses to be much greater than these, especially for the uniaxial FSCs, where the particles are brought into close proximity. The same qualitative effects should be observed in magnetic particle systems, and experience in electro- and magnetorheology indicates that the effect should be at least 10x larger. Thus we believe that uniaxial FSCs might be tenable candidates as artificial muscles.

Finally, experimental measurements of electrostriction should be made in very uniform, but large fields, to avoid the competing effect of dielectrophoresis - motion due to the force exerted on a dipole due to the gradient of the electric field. Dielectrophoresis and electrostriction are both quadratic in the field.

\section{Appendix A: The local field}

When working on this paper a number of questions arose about the cavity field, and this appendix is intended to explore some aspects of this. The treatment of the cavity field can be found in standard texts on electromagnetism, [14] and these comments are intended to elaborate on those treatments. In essence, the concept of the local or 
molecular field was created to account for the microscopic lumpiness of real dielectrics. The average field in a unit cell must be the macroscopic, or applied field $\mathbf{E}_{0}$, so that any line integral of the electric field through the dielectric, from one electrode to the other, equals the voltage drop. However, within a unit cell the actual electric field can vary considerably. For dipole lattices with cubic symmetry it is straightforward to show that at a dipole site (and at the center of the primitive unit cell - also a point of cubic symmetry) the field due to all of the dipoles in a cubical Lorentz cavity centered on the site is zero. Because the polarization is parallel to the field, the only contribution to the cavity field is due to the bound charge $\sigma_{p}=P$ on the $\mathrm{z}$ faces of the cube (field along $\mathrm{z}$ axis)

$$
\mathbf{E}_{L C}=\frac{\mathbf{P}}{4 \pi \varepsilon_{0}} \times 2 \int_{-1}^{1} d y \int_{-1}^{1} d x \frac{1}{\left(x^{2}+y^{2}+1\right)^{3 / 2}}=\frac{\mathbf{P}}{3 \varepsilon_{0}}
$$

Summing this with the applied field gives the total local field at a dipole site $\mathbf{E}_{l o c}=\frac{(\kappa+2)}{3} \mathbf{E}_{0}$, which can be considerably greater than the macroscopic field.

The Lorentz field is a clever bookkeeping construct that enables the correct computation of the local field. And admittedly, some cavity shapes have no associated fields. However, the local field can also be obtained without using cavity construct. Consider a cube of dielectric that is sandwiched between two infinite electrodes. The macroscopic field between the electrodes is then $E_{0} \hat{\mathbf{z}}$ both inside and outside the dielectric. The field at the dipole site which is at the center of this cube is produced by all charges in the system, i.e. all of the dipoles in the dielectric and the free charge on the electrodes. Because of the cubic symmetry, the dipoles produce no field at the central dipole site, so all of the bound charge, including that at the electrode surfaces comes to nought. The free charge on the electrodes consists of two contributions: outside the dielectric there is a free charge density $\sigma_{f}=\varepsilon_{0} E_{0}$, and above and below the dielectric the free charge density is just $\kappa \sigma_{f}$. Alternatively, one may sum the field from a free charge density of $\sigma_{f}$ over the entire electrodes, and an additional contribution of $(\kappa-1) \sigma_{f}$ above and below the dielectric. The local field at the dipole site is then 
$\mathbf{E}_{l o c}=\frac{E_{0}}{4 \pi} \times 2\left[\int_{-\infty}^{\infty} d y \int_{-\infty}^{\infty} d x \frac{1}{\left(x^{2}+y^{2}+1\right)^{3 / 2}}+\left.(\kappa-1) \int_{-1}^{1} d y \int_{-1}^{1} d x \frac{1}{\left(x^{2}+y^{2}+1\right)^{3 / 2}}\right|_{]}=E_{0}+\frac{(\kappa-1)}{3} E_{0}\right.$

and we see that the second integral, which is the sum over the excess free charge in the electrodes next to the dielectric, is exactly the Lorentz cavity field. Thus the result $\mathbf{E}_{l o c}=\frac{(\kappa+2)}{3} \mathbf{E}_{0}$ is obtained without invoking the cavity construct.

\section{Appendix B: Strain dependence of the cavity field}

The Lorentz cavity field can be obtained by integrating the electric field produced by the bound surface charge density, $\sigma_{p}=\mathbf{P} \cdot \hat{\mathbf{n}}$ where $\hat{\mathbf{n}}$ is a unit vector normal to the surface, over the walls of the cavity. For ease of computation, consider a cubical cavity of size $2 h$ on a side whose faces are normal to $\hat{x}, \hat{y}, \hat{z}$, subject this to small tensile strains, and compute the field at the center. Because the polarization is parallel to the $z$ axis, one only need integrate over the faces of the strained cube that are normal to the $\mathrm{z}$ axis. In terms of the tensile strains the integral is

$$
\mathbf{E}_{L C}=\frac{\mathbf{P}\left(1-\sum_{i} s_{i}\right)}{4 \pi \varepsilon_{0}} \times 2 \int_{-h\left(1+s_{2}\right)}^{h\left(1+s_{2}\right)} d y \int_{-h\left(1+s_{1}\right)}^{h\left(1+s_{1}\right)} d x \frac{h\left(1+s_{3}\right)}{\left(x^{2}+y^{2}+h^{2}\left(1+s_{3}\right)^{2}\right)^{3 / 2}}
$$

where the factor of 2 accounts for the two $z$ faces of the cube. After a change of variables, and recognizing the symmetry of the integral,

$$
\mathbf{E}_{L C}=\frac{\mathbf{P}}{4 \pi \varepsilon_{0}} \times 8 \int_{0}^{1} d y \int_{0}^{1} d x \frac{1}{\left(x^{2}\left(1+s_{1}\right)^{2}+y^{2}\left(1+s_{2}\right)^{2}+\left(1+s_{3}\right)^{2}\right)^{3 / 2}}
$$


where we have dropped terms $O\left(s^{2}\right)$. Evaluation of this integral at zero strain gives the standard result $\mathbf{E}_{L C}(\mathbf{0})=\mathbf{P} / 3 \varepsilon_{0}$. The cavity field can now be expanded to obtain the strain dependence to first order.

$$
\mathbf{E}_{L C}(\mathbf{s})=\mathbf{E}_{L C}(\mathbf{0})+\left.\mathbf{s} \cdot \nabla_{s} \mathbf{E}_{L C}(\mathbf{s})\right|_{\mathbf{s = 0}}
$$

Taking the derivatives of Eq. B. 2 with respect to strain gives

$$
\begin{aligned}
& \left.\frac{\partial \mathbf{E}_{L C}}{\partial s_{1}}\right|_{s_{1}=0}=\frac{-6 \mathbf{P}}{\pi \varepsilon_{0}} \int_{0}^{1} d x \int_{0}^{1} d y \frac{x^{2}}{\left(x^{2}+y^{2}+1\right)^{5 / 2}}=\frac{\mathbf{P}}{3 \varepsilon_{0}}\left(-1+\frac{\sqrt{3}}{\pi}\right) \\
& \left.\frac{\partial \mathbf{E}_{L C}}{\partial s_{3}}\right|_{s_{3}=0}=\frac{-6 \mathbf{P}}{\pi \varepsilon_{0}} \int_{0}^{1} d x \int_{0}^{1} d y \frac{1}{\left(x^{2}+y^{2}+1\right)^{5 / 2}}=\frac{\mathbf{P}}{3 \varepsilon_{0}}\left(-1-\frac{2 \sqrt{3}}{\pi}\right)
\end{aligned}
$$

Substituting these results into Eq. B.3 gives the cavity field in Eq. 6 . 


\section{Referenced Literature}

1. Proceedings of the 5 th International Conference on Electro-rheological fluids, Magneto-rheological Suspensions and Associated Technology, ed. by W. A. Bullough (World Scientific, Singapore, 1996)

2. J. E. Martin, R. A. Anderson, C. P. Tigges, Chapter 6 in Nanostructured Materials: Clusters, Composites, \& Thin Films, V. M. Shalaev and M. Moskovitz eds. ACS Symposium Series 679 (1996).

3. J. E. Martin, R. A. Anderson and C. P. Tigges, J. Chem. Phys. 1083765 (1998); ibid, J. Chem. Phys. 1087887 (1998).

4. J. E. Martin, C. P. Tigges, R. A. Anderson, "Enhanced Dielectric Standoff in FieldStructured Composites," submitted to Phys. Rev. E.

5. J. E. Martin, K. M. Hill, C. P. Tigges, "Field-Induced Optical Transmittance," to appear in Phys. Rev. E.

6. J. Lui, E. M. Lawrence, M. L. Ivey, G. A. Flores, J. Bibette, and J. Richard in Electrorheological Fluids: Mechanisms, Properties, Technology and Applications, ed. by R. Tao and G. D. Roy (World Scientific, Singapore, 1994) p. 172.

7. T. C. Halsey, R. A. Anderson, J. E. Martin, Inter. J. Mod. Phys. B 103019 (1996).

8. J. E. Martin and R. A. Anderson, J. Chem. Phys. 1044814 (1996).

9. M. Shibayama and T. Tanaka, Adv. Polym. Sci. 1091 (1993).

10. R. A. Anderson, Phys. Rev. B 33 1302, (1986).

11. R. A. Anderson, Langmuir 102917 (1994).

12. H. J. H. Clerkx and G. Bossis, Phys. Rev. E 482721 (1993).

13. L. C. Davis, J. Appl. Phys. 721334 (1992).

14. J. R. Reitz and F. J. Milford, Foundations of Electromagnetic Theory (Addison Wesley, Reading, MA, 1967), pp. 98-101. 


\section{Figure Captions}

1. Field-structured composites of particles structured by a uniaxial magnetic field (top), and by a biaxial magnetic field (bottom). Both samples consist of an Fe powder with a particle size of $4 \mu \mathrm{m}$ and at a concentration of $2.0 \mathrm{vol}$. \%. The magnification is $52 \mathrm{x}$.

2. The bistable potential well that an induced dipole feels when interacting with two neighboring field induced dipoles. This is the potential felt by the central dipole in a stretched chain of three polarized spheres of diameter $d$, with the centers of the end spheres fixed at 0 and $2 \mathrm{~d}$, aligned with the field.

3. An example of a simulated uniaxial FSC (top) and biaxial FSC, both at 30 vol. $\%$ particles 
Table I

Computed data for perfect lattices and for simulations of 10,000 particles in uniaxial and biaxial fields.

\begin{tabular}{|c|c|c|c|c|c|c|}
\hline$\phi$ & structure & $\psi_{2}$ & $\psi_{4}$ & $\kappa_{\text {eff }}^{\prime}(\beta=1)$ & $\lambda_{1}$ & $\lambda_{3}$ \\
\hline 0.523 & SC $\dagger$ & 0 & -0.294 & 4.30 & 0.505 & -1.01 \\
\hline 0.698 & BCT $\dagger$ & -0.032 & -0.012 & 9.82 & 0.167 & -0.427 \\
\hline 0.10 & random & 0 & 0 & 1.33 & 0.18 & -0.36 \\
\hline 0.10 & uniaxial & -0.271 & -0.189 & 1.84 & -0.28 & -4.85 \\
\hline 0.10 & biaxial & 0.493 & -0.395 & 1.16 & 5.26 & -0.66 \\
\hline 0.30 & random & 0 & 0 & 2.29 & 0.18 & -0.37 \\
\hline 0.30 & uniaxial & -0.185 & -0.137 & 3.73 & 0.09 & -1.42 \\
\hline 0.30 & biaxial & 0.331 & -0.304 & 1.66 & 1.39 & -0.58 \\
\hline 0.50 & random & 0 & 0 & 4.00 & 0.18 & -0.37 \\
\hline 0.50 & uniaxial & -0.083 & -0.078 & 5.49 & 0.18 & -0.69 \\
\hline 0.50 & biaxial & 0.131 & -0.181 & 2.97 & 0.54 & -0.56 \\
\hline
\end{tabular}

$\dagger \mathrm{SC}=$ simple cubic, $\dagger \dagger \mathrm{BCT}=$ body-centered tetragonal 
Table II

Computed data for perfect lattices and for simulations of 10,000 particles in uniaxial and biaxial fields.

\begin{tabular}{|c|c|c|c|c|c|c|}
\hline$\phi$ & structure & $\gamma_{31}$ & $\gamma_{33}$ & $\sigma_{1}^{*}$ & $\sigma_{3}^{*}$ & $\sigma_{3}^{*}-\sigma_{1}^{*}$ \\
\hline 0.523 & SC $\dagger$ & 0.33 & 4.17 & 2.88 & -22.2 & -25.1 \\
\hline 0.698 & BCT †† & 2.21 & 6.92 & -11.9 & -77.8 & -65.9 \\
\hline 0.10 & random & 0.69 & 0.31 & 0.97 & -1.75 & -2.7 \\
\hline 0.10 & uniaxial & 0.58 & 2.43 & 0.57 & -6.31 & -6.9 \\
\hline 0.10 & biaxial & 0.03 & 0.16 & 1.12 & -1.34 & -2.5 \\
\hline 0.30 & random & 0.68 & 1.08 & 0.73 & -4.76 & -5.5 \\
\hline 0.30 & uniaxial & 1.22 & 4.23 & -0.81 & -19.5 & -18.7 \\
\hline 0.30 & biaxial & 0.12 & 0.64 & 1.46 & -2.72 & -4.2 \\
\hline 0.50 & random & 1.09 & 2.33 & -0.35 & -13.3 & -13.0 \\
\hline 0.50 & uniaxial & 1.39 & 4.57 & -2.12 & -30.6 & -28.5 \\
\hline 0.50 & biaxial & 0.39 & 1.82 & 1.81 & -8.38 & -10.2 \\
\hline
\end{tabular}

$\nmid \mathrm{SC}=$ simple cubic, $\uparrow \nmid \mathrm{BCT}=$ body-centered tetragonal 

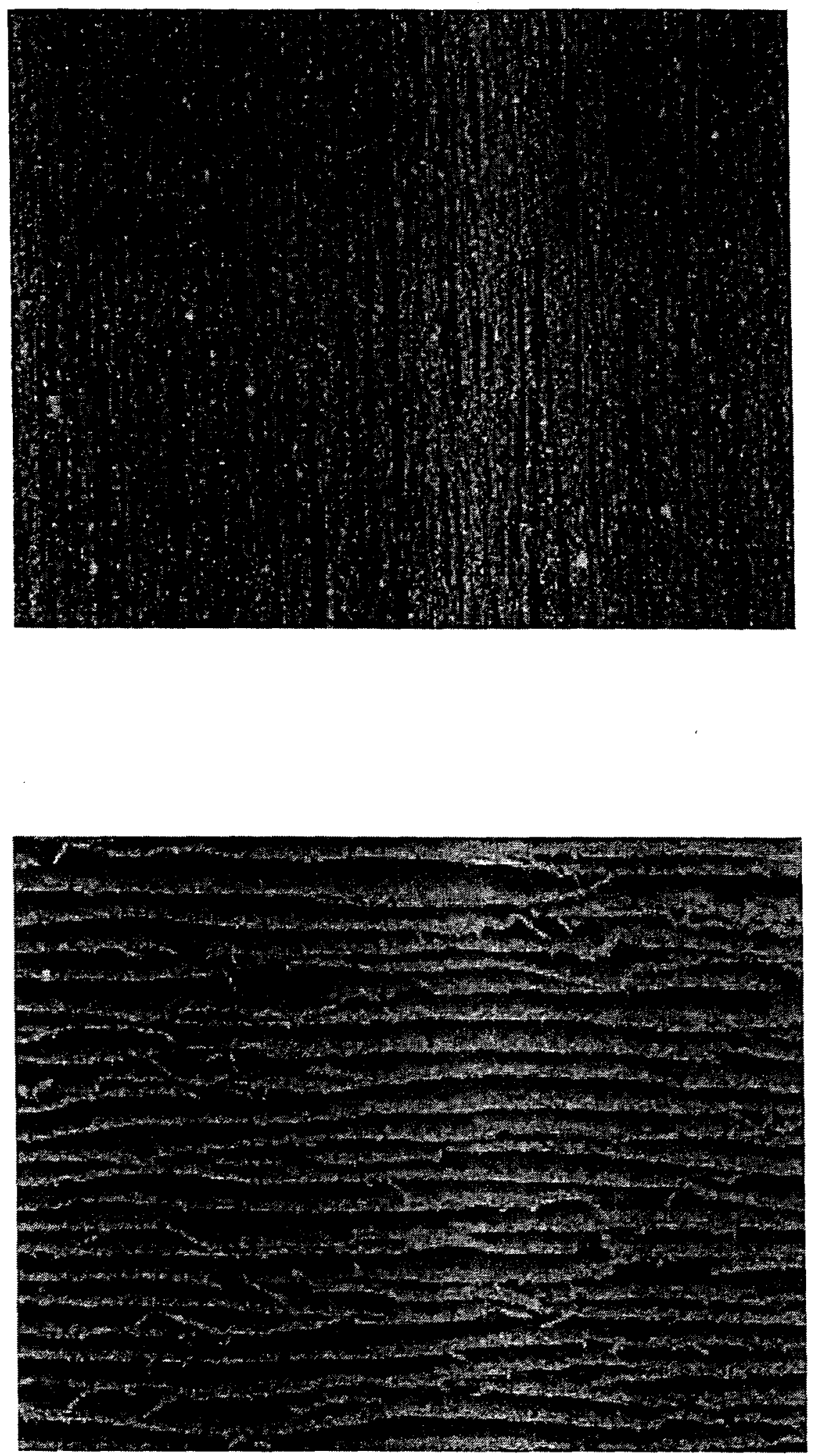

Fig. 1 Martin et.al. 


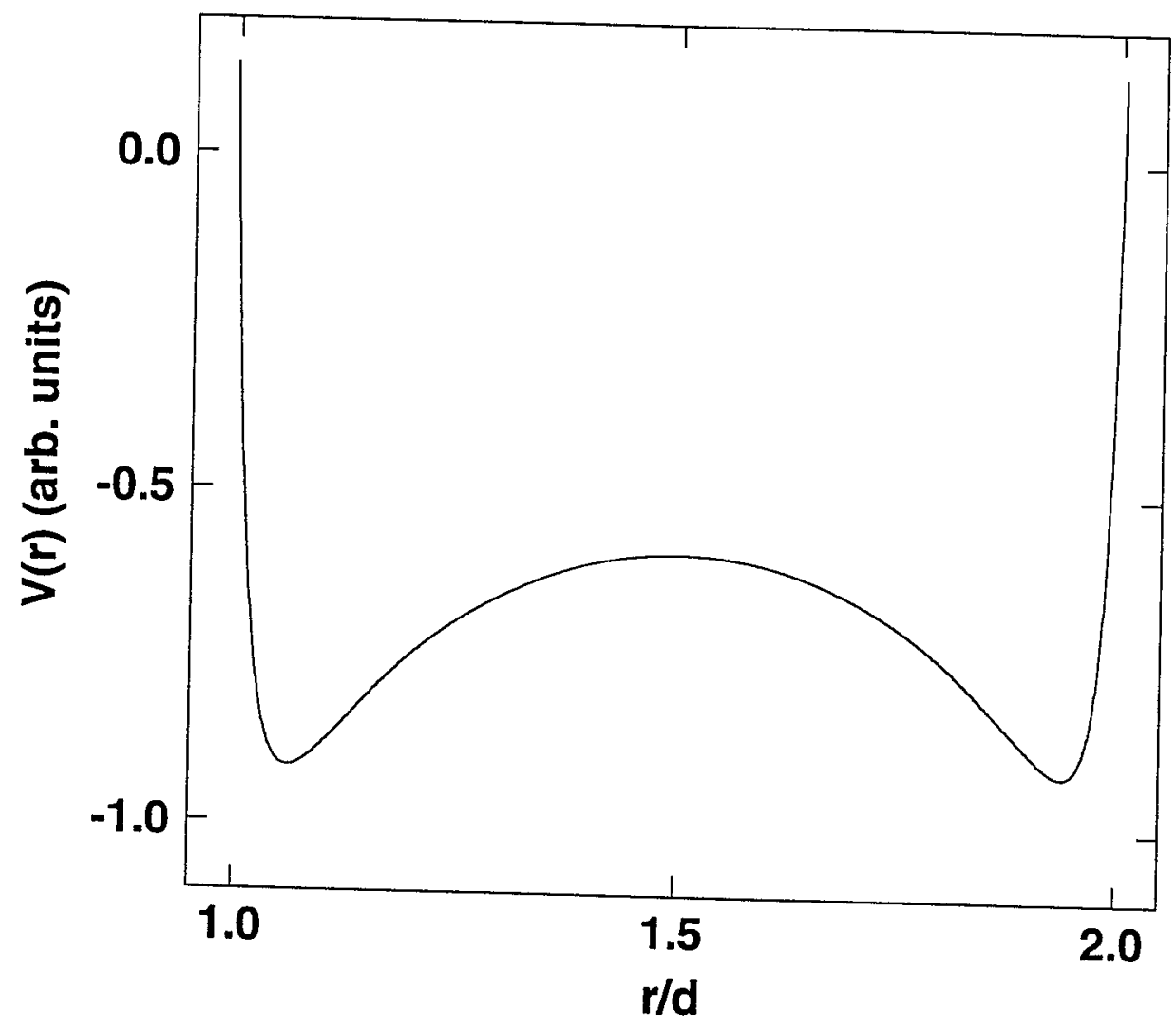

Fig.z Martin etal. 
$-4=$

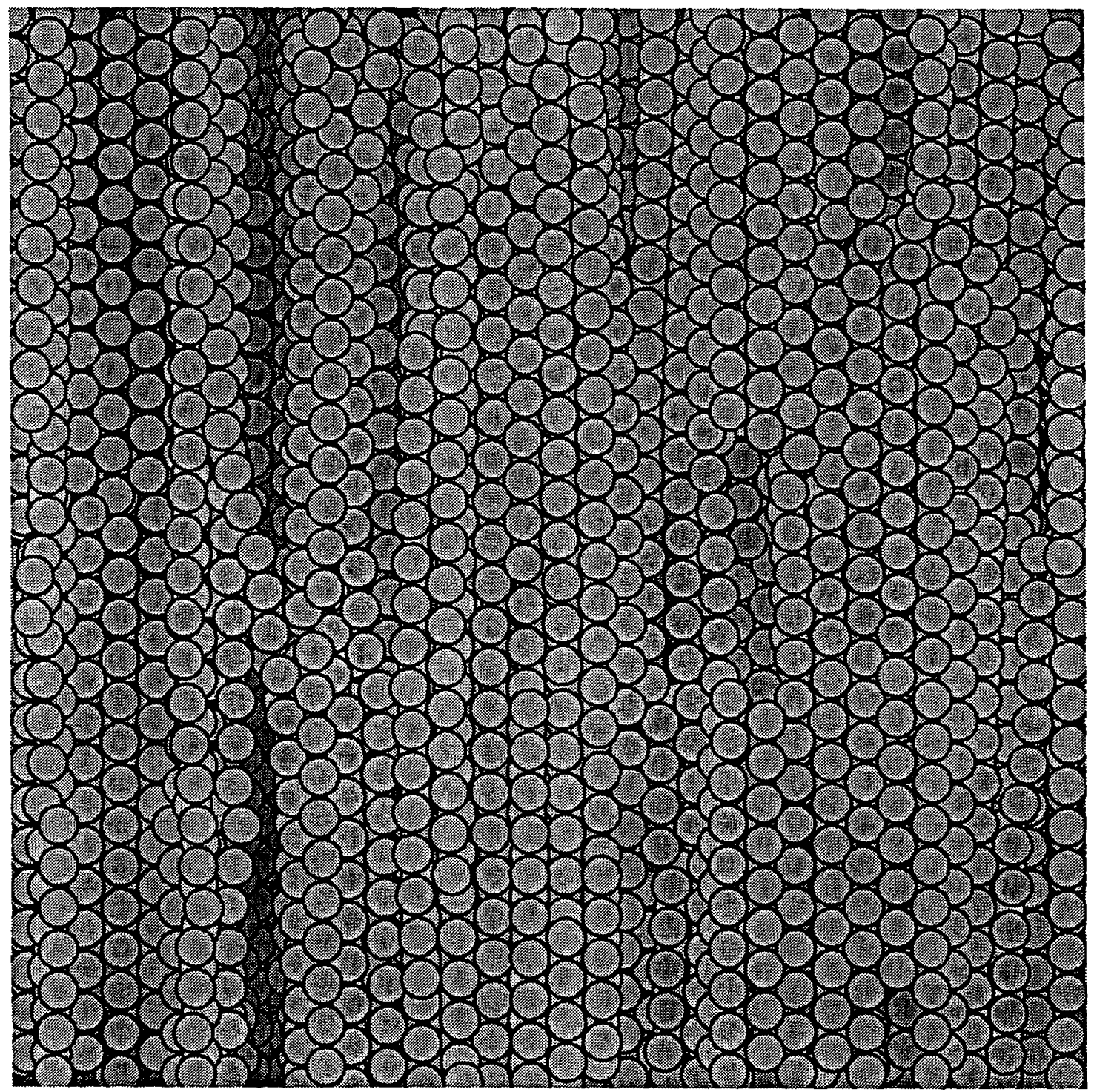

Fig. 3 (top) Martinetal 


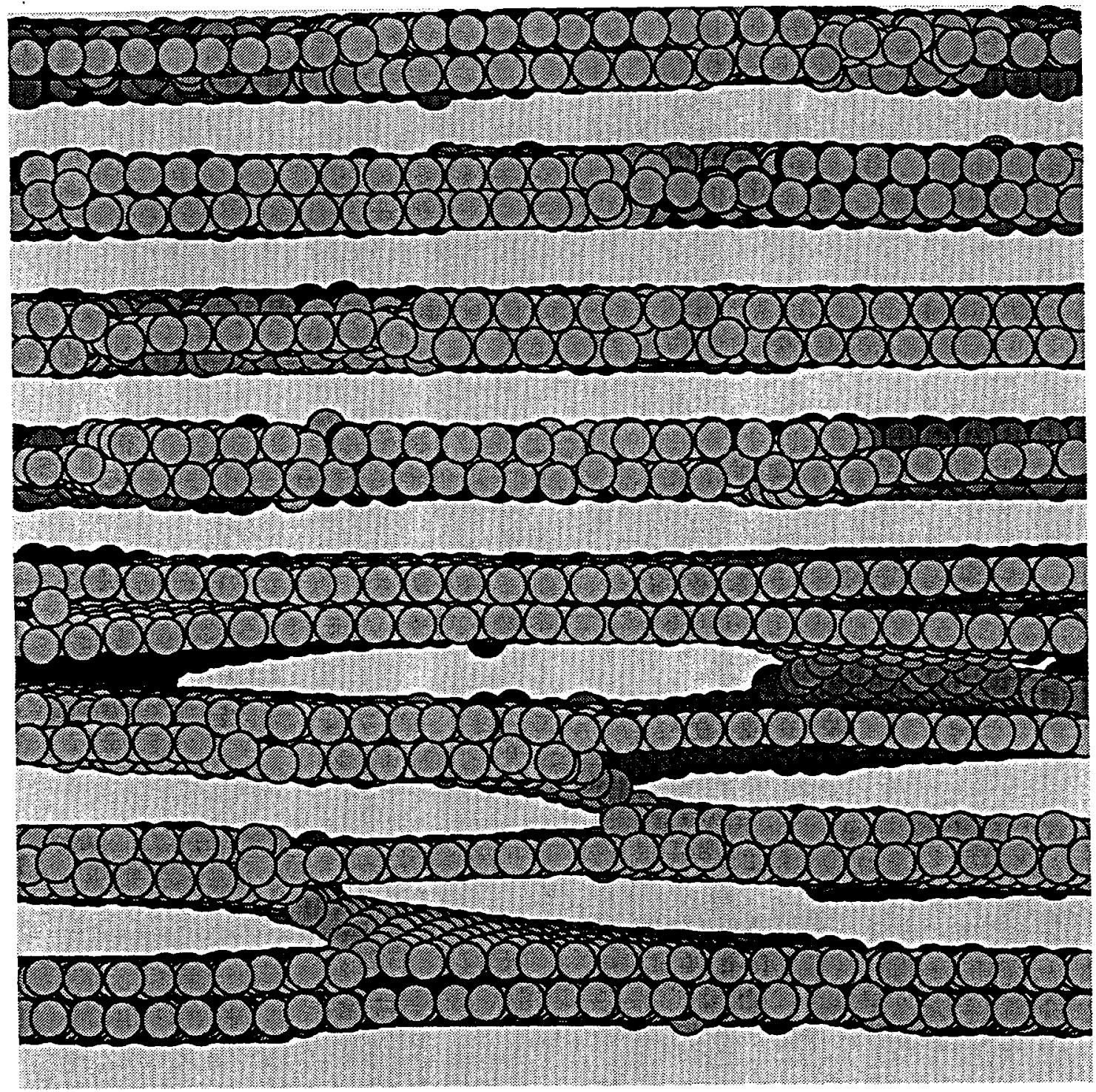

Fig. 3 (bottom) Martin etal. 